\title{
Pancreatic Diseases Associated with Pancreas Divisum
}

\author{
Kensuke Takuma ${ }^{a}$ Terumi Kamisawa $^{a}$ Taku Tabata ${ }^{a}$ Naoto Egawa ${ }^{a}$ \\ Yoshinori Igarashi ${ }^{\text {b }}$

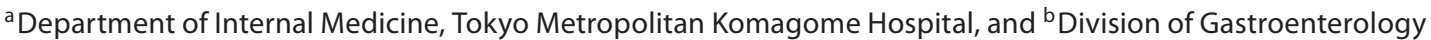 \\ and Hepatology, Department of Internal Medicine, Toho University School of Medicine, Tokyo, Japan
}

\section{Key Words}

Acute pancreatitis - Alcohol intake $\cdot$ Chronic pancreatitis •

Pancreas cancer $\cdot$ Pancreas divisum

\begin{abstract}
Aim: To clarify the features of acute or chronic pancreatitis and pancreatic tumors associated with complete and incomplete pancreas divisum. Methods: Clinical features of pancreatic diseases associated with complete $(n=54)$ and incomplete $(n=50)$ pancreas divisum were examined. Results: Acute and chronic pancreatitis occurred more frequently in patients with complete pancreas divisum (22.2\%: 12/54, and 18.5\%: 10/54, respectively) than in controls (5.6\%: 183/3,246, and 4.9\%: 159/3,246, respectively; $\mathrm{p}<0.01)$. In 8 patients with chronic pancreatitis, the dorsal pancreatic duct was irregularly dilated from the orifice to the tail, whereas the ventral pancreatic duct was not dilated (isolated dorsal pancreatitis). None of the 12 complete pancreas divisum patients with acute pancreatitis consumed heavy or moderate amounts of alcohol. Although 6 of 10 patients with chronic pancreatitis consumed heavy or moderate amounts of alcohol, 31 of 32 patients with this malformation who did not exhibit pancreatitis consumed little or no alcohol. Chronic pancreatitis occurred more frequently in patients with incomplete pancreas divisum (18.0\%: 9/50) than in controls (4.9\%: 159/3,246; $\mathrm{p}<0.01$ ). Of 54 patients with complete pancreas divisum, 6 patients (11.1\%) were associated with pancreatic tumor. All tumors developed from the dorsal pancreas of pancreas di-
\end{abstract}

visum. Conclusions: Complete pancreas divisum can be the sole etiology of acute or chronic pancreatitis. The presence of another factor, such as alcohol abuse, may be required for chronic pancreatitis to develop in some patients with complete or incomplete pancreas divisum. In complete pancreas divisum, the dorsal pancreatic duct might be a factor that promotes oncogenesis.

Copyright $\odot 2010$ S. Karger AG, Base

\section{Introduction}

The human pancreas develops embryologically from the fusion of the ventral and dorsal buds. Under normal conditions, the superior branch of the ventral pancreatic duct fuses with the dorsal pancreatic duct and participates in the formation of the main pancreatic duct $[1,2]$. In the embryo, the accessory pancreatic duct is the main drainage duct of the dorsal pancreas and enters the duodenum at the minor duodenal papilla. As development progresses, the duct of the dorsal bud undergoes varying degrees of atrophy at the duodenal end, finally forming the adult configuration of the duct [1-3].

Pancreas divisum is a common congenital anomaly of the pancreas, which results from an abnormal fusion of the ventral and dorsal pancreatic ducts during fetal development. It is divided into complete and incomplete pancreas divisum. Complete pancreas divisum is defined as a completely separate pancreatic duct system. Incom-

\section{KARGER}

Fax +4161306 1234

E-Mail karger@karger.ch

www.karger.com
(C) 2010 S. Karger AG, Basel

$0253-4886 / 10 / 0272-0144 \$ 26.00 / 0$

Accessible online at:

www.karger.com/dsu
Terumi Kamisawa, MD, PhD

Department of Internal Medicine, Tokyo Metropolitan Komagome Hospital

3-18-22 Honkomagome, Bunkyo-ku

Tokyo 113-8677 (Japan)

Tel. +81 33823 2101, Fax +8133824 1552, E-Mail kamisawa @ cick.jp 
Table 1. Prevalence of acute and chronic pancreatitis associated with complete or incomplete pancreas divisum and controls

\begin{tabular}{|c|c|c|c|c|}
\hline & \multicolumn{4}{|l|}{ Patients } \\
\hline & $\begin{array}{l}\text { acute } \\
\text { pancreatitis }\end{array}$ & $\mathrm{p}$ values ${ }^{1}$ & $\begin{array}{l}\text { chronic } \\
\text { pancreatitis }\end{array}$ & p values ${ }^{1}$ \\
\hline Complete PD $(\mathrm{n}=54)$ & $12(22.2 \%)$ & $<0.01$ & $10(18.5 \%)$ & $<0.01$ \\
\hline Incomplete PD $(\mathrm{n}=50)$ & $3(6.0 \%)$ & 0.75 & $9(18.0 \%)$ & $<0.01$ \\
\hline Controls $(n=3,246)$ & $183(5.6 \%)$ & & $159(4.9 \%)$ & \\
\hline Total $(\mathrm{n}=3,350)$ & $198(5.9 \%)$ & & $178(5.3 \%)$ & \\
\hline
\end{tabular}

$\mathrm{PD}=$ Pancreas divisum. ${ }^{1}$ Compared with controls. plete pancreas divisum is an anatomic variation that has an inadequate communication, usually an extremely small branch, between the ventral and dorsal pancreatic ducts [4-6].

Pancreas divisum is sometimes associated with acute or chronic pancreatitis [4-9]. However, some patients have a history of alcohol consumption, which obscures the real contribution of the anomaly to pancreatitis. An association between pancreas divisum and pancreatic tumors has been reported by some researchers [10-13]. To clarify the clinical significance of pancreas divisum, we investigated retrospectively the prevalence of pancreatitis and pancreatic tumors in pancreas divisum.

\section{Patients and Methods}

\section{Patients}

We have documented the anatomy of the pancreatic duct system using endoscopic retrograde pancreatography via the minor duodenal papilla as well as the major duodenal papilla at the Tokyo Metropolitan Komagome Hospital.

Complete pancreas divisum was diagnosed when filling of the entire dorsal pancreatic duct from the tail to the anterior part of the head was observed without communication with the short ventral pancreatic duct on cannulation of the minor duodenal papilla. When the short ventral pancreatic duct was seen on ventral pancreatography but no dorsal pancreatography could be obtained by cannulation from the minor duodenal papilla, diagnosis of complete pancreas divisum was confirmed from the magnetic resonance cholangiography findings of a dominant dorsal pancreatic duct crossing the lower bile duct and emptying into the duodenum without communicating with the ventral pancreatic duct. Incomplete pancreas divisum was diagnosed when the ventral and dorsal pancreatic ducts ran parallel to each other and were inadequately communicated, usually with an extremely small branch or a branch from the dorsal pancreatic duct running toward the ventral pancreatic duct in a roundabout $[4,6,14]$.

Of 3,350 cases with successful pancreatography, complete pancreas divisum was identified in 54 patients (1.6\%). Incomplete pancreas divisum was diagnosed in 50 patients (1.4\%). Endoscop- ic retrograde pancreatography was performed in 198 patients with acute pancreatitis after a bout of inflammation and in 178 patients with chronic pancreatitis. The prevalence of acute and chronic pancreatitis associated with complete or incomplete pancreas divisum was compared with that of 3,246 cases without complete or incomplete pancreas divisum.

\section{Clinical Analysis}

History of alcohol abuse and associated pancreatic diseases of acute or chronic pancreatitis was compared between patients with complete and incomplete pancreas divisum. The amount of alcohol consumed by patients was evaluated by follow-up contact or chart review. The degree of alcohol consumption was classified into heavy: ( $\geq 100 \mathrm{~g}$ alcohol/day for over 10 years), moderate: ( $<100 \mathrm{~g} /$ day but $\geq 30 \mathrm{~g} /$ day), mild (a little and occasionally), and none. The diagnosis of acute pancreatitis was based on episodes of abdominal pain with twice-normal elevations in serum amylase or lipase, typical changes in ultrasonography or computed tomography (CT), and absence of ductal changes characteristic of chronic pancreatitis. The diagnosis of chronic pancreatitis was based on either the existence of pancreatic stones visible on plain films of the abdomen or CT, or characteristic ductal changes on endoscopic retrograde cholangiopancreatography (ERCP) [9].

The prevalence of pancreatic cancers and intraductal papillary mucinous neoplasms associated with pancreas divisum was examined, and diagnoses were based on ERCP, ultrasound, CT, cytology, and surgical findings.

\section{Statistical Analysis}

Data were statistically compared with those in controls using Fisher's exact test. $\mathrm{p}<0.05$ was considered statistically significant.

\section{Results}

\section{Pancreatitis with Complete Pancreas Divisum}

Acute and chronic pancreatitis occurred more frequently in patients with complete pancreas divisum (22.2\%: $12 / 54$, and 18.5\%: $10 / 54$, respectively) than in controls (5.6\%: 183/3,246, and 4.9\%: 159/3,246, respectively; $\mathrm{p}<0.01$; table 1$)$. The degree of acute pancreatitis was mild with this malformation, but attacks were relaps- 
Table 2. Relationship between alcohol intake and occurrence of pancreatitis in patients with complete or incomplete pancreas divisum

\begin{tabular}{llll}
\hline & & $\begin{array}{l}\text { Alcohol intake } \\
++ \text { to }+\end{array}$ & $\begin{array}{c}\text { Alcohol intake } \\
\pm \text { to }-\end{array}$ \\
\hline $\begin{array}{c}\text { Complete pancreas divisum } \\
\text { Associated pancreatitis }\end{array}$ & 54 & & \\
$\quad$ Acute pancreatitis & 12 & 0 & $12\{8\}$ \\
Chronic pancreatitis & 10 & $6(4)$ & $4(4)\{2\}$ \\
$\quad$ No associated pancreatitis & 32 & 1 & 31 \\
Incomplete pancreas divisum & 50 & & $3\{1\}$ \\
Associated pancreatitis & & & $2(2)$ \\
$\quad$ Acute pancreatitis & 4 & 1 & 35 \\
$\quad$ Chronic pancreatitis & 9 & 7 & \\
No associated pancreatitis & 37 & 2 & \\
\hline
\end{tabular}

(Number of cases of isolated dorsal pancreatitis) and number of cases of relapsing pancreatitis\}. ing in 8 patients. In 8 patients with chronic pancreatitis, the dorsal pancreatic duct was irregularly dilated from the orifice to the tail, whereas the ventral pancreatic duct was not dilated (isolated dorsal pancreatitis; table 2). Pancreatic stones and cysts were detected in 3 patients, respectively. Two relapsing pancreatitis patients who underwent endoscopic minor papilla sphincterotomy achieved improvement, but restenosis occurred in $1 \mathrm{pa}-$ tient.

None of the 12 patients with acute pancreatitis found to have this malformation consumed heavy or moderate amounts of alcohol. Although 6 of 10 patients with chronic pancreatitis consumed heavy or moderate amounts of alcohol, 31 of 32 patients with this malformation who did not exhibit pancreatitis consumed little or no alcohol (table 2).

\section{Pancreatitis with Incomplete Pancreas Divisum}

Acute pancreatitis was identified in 3 (6.0\%) of 50 patients with incomplete pancreas divisum. Chronic pancreatitis occurred more frequently in patients with incomplete pancreas divisum (18.0\%: 9/50) than in controls (4.9\%: 159/3,246; $p<0.01$; table 1 ). In the 10 patients with incomplete divisum who abused alcohol, 1 patient had acute pancreatitis and 7 patients had chronic pancreatitis of the entire organ. Thirty-five of 37 patients with this malformation who did not exhibit pancreatitis consumed little or no alcohol (table 2).

\section{Pancreatic Tumors with Pancreas Divisum}

Of 54 patients with complete pancreas divisum, 6 patients (11\%) were associated with pancreatic tumor: pancreatic carcinoma $(n=5)$ and intraductal papillary muci- nous neoplasm $(\mathrm{n}=1)$. Three were male and 3 female, and average patient age was 72.3 years. Tumors were located in the pancreas head $(n=3)$, body $(n=2)$, and tail $(n=1)$. Histologically, the associated pancreatic carcinoma was adenocarcinoma. The ventral pancreatic duct was not affected by the tumor in any case. Although there was no clinical evidence of chronic dorsal pancreatitis, the dorsal pancreatic ducts of all cases were slightly dilated (maximum diameter: 4-5 mm). All tumors developed from the dorsal pancreas of pancreas divisum.

\section{Discussion}

Failure of the ventral and dorsal pancreatic duct to unite results in the anatomic variant pancreas divisum. In pancreas divisum, the dorsal pancreatic duct becomes the main pancreatic duct and drains most of the pancreatic fluid through the minor duodenal papilla. As the minor duodenal papilla is substantially smaller than the major duodenal papilla, a larger secretory capacity might, presumably, place a significant load on the minor duodenal papilla $[4,14]$. Considerable controversy exists in the literature regarding whether pancreas divisum is etiologically related to pancreatitis. Arguments for pancreas divisum being involved in the pathogenesis of pancreatitis are based on four interrelated principles: (1) an increased incidence (50.0 or $25.6 \%)$ of pancreas divisum in idiopathic acute relapsing pancreatitis in ERCP series $[9,15]$; (2) a logical explanation based on the concept of relative outflow obstruction of pancreatic juice through the minor duodenal papilla derived from provocative tests [5] or due to increased dorsal ductal pressure compared with 
normal controls measured using endoscopic manometry [16]; (3) isolated dorsal pancreatitis, as shown by irregular dilatation apparent on dorsal pancreatography alone [17, 18], and (4) improvement after endoscopic or surgical procedures that open the minor duodenal papilla $[5,14$, 19]. Against these are the counter arguments that question the evidence for each proposal: (1) fewer than $5 \%$ of the population with pancreas divisum develop pancreatic symptoms; (2) many patients with pancreas divisum who develop pancreatitis are not young; (3) the association between pancreatitis and pancreas divisum is questionable since ERCP series reflect referral populations that vary from center to center $[20,21]$; (4) little direct evidence indicates outflow obstruction from the dorsal pancreatic duct during physiological pancreatic secretion and even the non-physiological secretin ultrasound test has failed to give consistent results [22], and (5) responses to endoscopic and surgical management are variable and unpredictable $[23,24]$.

In this series, acute pancreatitis occurred in $22.2 \%$ of the patients with complete pancreas divisum, and the rate was significantly higher than in controls. As the 12 patients consumed little or no alcohol, we believe that complete pancreas divisum was the etiology of acute pancreatitis in these cases. Although in 6 patients chronic pancreatitis was associated with alcohol consumption, the dorsal pancreatic duct showed more irregular alterations than the ventral pancreatic duct in 5 of the 6 patients. Again, in the 4 patients with little or no alcohol intake, complete pancreas divisum was thought to be the sole etiology of chronic pancreatitis. In 8 patients, the dorsal pancreatic duct was irregularly dilated from the orifice to the tail, whereas the ventral pancreatic duct was not dilated (isolated dorsal pancreatitis). The dilatation of the entire dorsal pancreatic duct proximal to the minor duodenal papilla suggests that dysfunction of the minor papilla could cause obstructive pancreatitis. However, as most patients with complete pancreas divisum but mini- mal alcohol intake did not develop pancreatitis, the presence of another factor, such as alcohol abuse, may be required for chronic pancreatitis to develop in some individuals.

The association between acute pancreatitis and incomplete pancreas divisum was not significantly stronger than in controls. Prevalence of chronic pancreatitis associated with complete and incomplete pancreas divisum was similar, but isolated dorsal pancreatitis was less frequent in patients with incomplete pancreas divisum. Although some cases of incomplete pancreas divisum have a dorsal pancreatic duct-dominant physiological status, the precise pathophysiology of incomplete pancreas divisum may be different from that of complete pancreas divisum.

In the present study, pancreatic carcinoma occurred in 5 (9.2\%) of 54 patients with pancreas divisum, and all pancreatic carcinomas developed in the dorsal pancreas. In one of them, microscopic findings of periductal and interlobular fibrosis detected in the non-carcinomatous pancreas resected implied that chronic dorsal pancreatitis associated with pancreas divisum preceded the carcinoma. According to Okada et al. [11], pancreatic carcinoma developed in the dorsal pancreas in 24 of 25 cases of pancreatic carcinoma associated with pancreas divisum. Nishino et al. [12] also reported that pancreatic carcinoma developed in the dorsal pancreas in 11 of 12 cases with pancreas divisum. These findings suggest that longstanding dorsal duct obstruction caused by the relative stenosis of the minor duodenal papilla might be a factor that promotes pancreatic carcinoma $[10,13]$.

In conclusion, complete pancreas divisum can be the sole etiology of acute or chronic pancreatitis. The presence of another factor, such as alcohol abuse, may be required for chronic pancreatitis to develop in some individuals with complete or incomplete pancreas divisum. In complete pancreas divisum, dorsal pancreatic duct obstruction might be a factor that promotes oncogenesis.
References

Pancreatic Diseases and Pancreas Divisum
1 Adda G, Hannoun L, Loygue J: Development of the human pancreas: variations and pathology. A tentative classification. Anat Clin 1984;5:275-283.

-2 Kamisawa T, Koike M, Okamoto A: Embryology of the pancreatic duct system. Digestion 1999;60:161-165.

3 Kamisawa T, Tabata I, Tajima T, Tushima K, Yoshida Y: Patency of the human accessory pancreatic duct determined by dye-injection endoscopic retrograde pancreatography. Digestion 1997;58:78-82.

\footnotetext{
4 Kamisawa T: Clinical significance of the minor duodenal papilla and accessory pancreatic duct. J Gastroenterol 2004;39:605-615.

5 Warshaw AL, Simeone JF, Schapiro RH, Flavin-Warshaw B: Evaluation and treatment of the dorsal duct syndrome (pancreas divisum redefined). Am J Surg 1990;159:59-66.

$\checkmark 6$ Kamisawa T, Egawa N, Tsuruta K, Okamoto A, Matsukawa M: Pancreatitis associated with congenital abnormalities of the pancreaticobiliary system. Hepatogastroenterology 2005;52:223-229.
} 
7 Lehman GA, Sherman S, Nisi R, Hawes RH: Pancreas divisum: results of minor papilla sphincterotomy. Gastrointest Endosc 1993; 39:1-8.

-8 Toki F, Kozu T, Yoshida K, Nishino T, Tadokoro $\mathrm{H}$ : Pancreatogram of unfused pancreatic duct system. Dig Endosc 1994;6:152-157.

$>9$ Bernard JP, Sahel J, Giovannini M, Sahels H: Pancreas divisum is a probable cause of acute pancreatitis: a report of 137 cases. Pancreas 1990;5:248-254.

10 Traverso LW, Kozarek RA, Simpson T, Galagan KA: Pancreatic duct obstruction as a potential etiology of pancreatic adenocarcinoma: a clue from pancreas divisum. Am J Gastroenterol 1993;88:117-119.

11 Okada J, Uesaka K, Morimoto T, Kodera Y, Torii A, Hirai T, Yasui K, Yamamura Y, Kato T, Kito T: A case of pancreatic cancer of the dorsal pancreas associated with pancreas divisum. Jpn J Gastroenterol Surg 1996;29: 843-847.
12 Nishino T, Toki F, Oi I, Oyama H, Hatori T, Shiratori K: Prevalence of pancreatic and biliary tract tumors in pancreas divisum. J Gastroenterol 2006;41:1088-1093.

13 Kamisawa T, Yoshiike M, Egawa N, Tsuruta K, Okamoto A, Funata N: Pancreatic tumor associated with pancreas divisum. J Gastroenterol Hepatol 2005;20:915-918.

14 Kamisawa T: Endoscopic approach to the minor duodenal papilla: special emphasis on endoscopic management on pancreas divisum. Dig Endosc 2006;18:252-255.

15 Cotton PB: Congenital anomaly of pancreas divisum as cause of obstructive pain and pancreatitis. Gut 1980;21:105-114.

$>16$ Satterfield ST, McCarthy JH, Geenen JE, Hogan WJ, Venu RP, Venu RP, Dodds WJ, Johnson GK: Clinical experience in 82 patients with pancreas divisum: preliminary results of manometry and endoscopic therapy. Pancreas 1988;3:248-253.

17 Blair AJ, Russell CG, Cotton PB: Resection for pancreatitis in patients with pancreas divisum. Ann Surg 1984;200:590-594.

18 Lindstroom E, von Schenk H, Ihse I: Pancreatic exocrine and endocrine function in patients with pancreas divisum and abdominal pain. Int J Pancreatol 1990;6:17-24.
19 Siegel JH, Ben-Zvi JS, Pullano W, Cooperman A: Effectiveness of endoscopic drainage for pancreas divisum: endoscopic and surgical results in 31 patients. Endoscopy 1990;22: 129-133.

20 Delhaye M, Engelholm L, Cremer M: Pancreas divisum: congenital anatomic variant or anatomy? Contribution of endoscopic retrograde dorsal pancreatography. Gastroenterology 1985;89:951-958.

21 Burtin P, Person B, Charneau J, Person B, Pujol P, Valentin S, D'Anbigny N, Joubaud F, Boyer J: Pancreas divisum: a coincidental association? Endoscopy 1991;23:55-58.

22 Lowes JR, Lees WR, Cotton PB: Pancreatic duct dilatation after secretin stimulation in patients with pancreas divisum. Pancreas 1989;4:371-374.

23 Jacooks MA, ReMine SG, Carmichael DH: Difficulties in the diagnosis and treatment of pancreas divisum. Arch Surg 1984;119: 1088-1091.

24 Varshney S, Johnson CD: Pancreas divisum. Int J Pancreatol 1999;25:135-141. 